\title{
NORMAL DERIVATIONS IN NORM IDEALS
}

\author{
FUAD KITTANEH
}

(Communicated by Palle E. T. Jorgensen)

\begin{abstract}
We establish the orthogonality of the range and the kernel of a normal derivation with respect to the unitarily invariant norms associated with norm ideals of operators. Related orthogonality results for certain nonnormal derivations are also given.
\end{abstract}

\section{INTRODUCTION}

Let $B(H)$ denote the algebra of all bounded linear operators on an infinitedimensional complex separable Hilbert space $H$. For operators $A, B$ in $B(H)$, the generalized derivation $\delta_{A, B}$ as an operator on $B(H)$ is defined by

$$
\delta_{A, B}(X)=A X-X B \text { for all } X \in B(H) .
$$

When $A=B$, we simply write $\delta_{A}$ for $\delta_{A, A}$. If $N$ is a normal operator in $B(H)$, then $\delta_{N}$ is said to be a normal derivation.

In his investigation of normal derivations, Anderson [1, Theorem 1.7] proved that if $N$ and $S$ are operators in $B(H)$ such that $N$ is normal and $N S=S N$, then for all $X \in B(H)$

$$
\left\|\delta_{N}(X)+S\right\| \geq\|S\|,
$$

where $\|\cdot\|$ is the usual operator norm. Thus in the sense of [1, Definition 1.2], inequality (2) says that the range of $\delta_{N}$ is orthogonal to the kernel of $\delta_{N}$, which is just the commutant $\{N\}^{\prime}$ of $N$.

It has been shown in [11, Theorem 1] that if $N$ and $S$ are operators in $B(H)$ such that $N$ is normal, $S$ is a Hilbert-Schmidt operator, and $S \in\{N\}^{\prime}$, then for all $X \in B(H)$

$$
\left\|\delta_{N}(X)+S\right\|_{2}^{2}=\left\|\delta_{N}(X)\right\|_{2}^{2}+\|S\|_{2}^{2},
$$

where $\|\cdot\|_{2}$ is the Hilbert-Schmidt norm. Thus in the usual Hilbert space sense, the Hilbert-Schmidt operators in the range of $\delta_{N}$ are orthogonal to those in the kernel of $\delta_{N}$.

Received by the editors July 22, 1993 and, in revised form, September 28, 1993.

1991 Mathematics Subject Classification. Primary 47A30, 47B10, 47B15, 47B20, 47B47; Secondary 46B20.

Key words and phrases. Normal derivation, nonnormal derivation, norm ideal, unitarily invariant norm, orthogonality results for derivations. 
It has also been shown recently in [12, Theorem 3.2] that if $N$ and $S$ are operators in $B(H)$ such that $N$ is normal and $S$ belongs to some Schatten $p$-class $C_{p}$ with $1 \leq p \leq \infty$ and $S \in\{N\}^{\prime}$, then for all $X \in B(H)$

$$
\left\|\delta_{N}(X)+S\right\|_{p} \geq\|S\|_{p} .
$$

The usual operator norm, the Hilbert-Schmidt norm, and the Schatten $p$ norms are only examples of a large family of unitarily invariant (or symmetric) norms on $B(H)$.

The purpose of this paper is to investigate the orthogonality of the range and the kernel of a normal derivation with respect to the wider class of unitarily invariant norms on $B(H)$. Derivations induced by certain nonnormal operators will also be discussed.

In $\S 2$ we will use a completely different analysis to extend (4) to all unitarily invariant norms defined on norm ideals of compact operators in $B(H)$. Extensions of this result to certain nonnormal operators will be the main theme of $\S 3$, in which we will treat derivations of the form $\delta_{A, B}$, where $A$ is a dominant operator and $B^{*}$ is $M$-hyponormal. Moreover we will discuss the validity of (2) for various classes of derivations at the expense of requiring that $S$ is normal. A relevant example will also be presented.

Recall that each unitarily invariant norm $\||\cdot|\| \mid$ is defined on a natural subclass $J_{\|\| \cdot \| \mid}$ of $B(H)$ called the norm ideal associated with the norm $\|\mid \cdot\|$ and satisfies the invariance property $\|U A V\|\|=\| A\|\|$ for all $A \in J_{\|\| \cdot\|\|}$ and for all unitary operators $U, V \in B(H)$. While the usual operator norm $\|\cdot\|$ is defined on all of $B(H)$, the other unitarily invariant norms are defined on norm ideals contained in the ideal of compact operators in $B(H)$. Given any compact operator $A \in B(H)$, denote by $s_{1}(A) \geq s_{2}(A) \geq \cdots$ the singular values of $A$, i.e., the eigenvalues of $|A|=\left(A^{*} A\right)^{1 / 2}$. There is a one-to-one correspondence between symmetric gauge functions defined on sequences of real numbers and unitarily invariant norms defined on norm ideals of operators. More precisely, if $\||\cdot \||$ is a unitarily invariant norm, then there is a unique symmetric gauge function $\Phi$ such that

$$
\||| A||=\Phi\left(\left\{s_{j}(A)\right\}\right)
$$

for all $A \in J_{\|\| \cdot\|\|}$.

For $1 \leq p \leq \infty$, define

$$
\|A\|_{p}=\left(\sum_{j} s_{j}^{p}(A)\right)^{1 / p},
$$

where, by convention, $\|A\|_{\infty}=s_{1}(A)$ is the usual operator norm of the compact operator $A$. These unitarily invariant norms are the well-known Schatten $p$ norms associated with the Schatten $p$-classes $C_{p}, 1 \leq p \leq \infty$. Hence $C_{1}, C_{2}$, and $C_{\infty}$ are the trace class, the Hilbert-Schmidt class, and the class of compact operators, respectively. For good accounts on the theory of norm ideals and their associated unitarily invariant norms, the reader is referred to [9], [13], or [14] (see also [2] and references therein).

\section{NORMAL DERIVATIONS}

In this section we present our main result of this paper. This result asserts that if $N$ is a normal operator in $B(H)$, then with respect to any unitarily 
invariant norm $\||\cdot|\| \mid, \operatorname{ran} \delta_{N} \cap J_{\||| \cdot||}$ is orthogonal to $\operatorname{ker} \delta_{N} \cap J_{\||| \cdot||}$, where $\operatorname{ran} \delta_{N}$ and $\operatorname{ker} \delta_{N}$ are the range and the kernel of $\delta_{N}$, respectively.

To accomplish our goal we need two lemmas.

Lemma 1. Let $N \in B(H)$ be diagonal (normal with pure point spectrum), $S \in$ $\{N\}^{\prime}$, and $X \in B(H)$. If $\delta_{N}(X)+S \in J_{\||\cdot|\|}$, then $S \in J_{\|\| \cdot \| \mid}$ and

$$
\left\|\delta_{N}(X)+S|\|\geq\|||S|\right\| \text {. }
$$

Proof. Let $N$ have the distinct eigenvalues $\lambda_{1}, \lambda_{2}, \ldots$. Then, with respect to the decomposition $H=\bigoplus_{j=1}^{\infty} \operatorname{ker}\left(N-\lambda_{j}\right), N$ has the operator matrix representation

$$
N=\left[\begin{array}{lll}
\lambda_{1} & & 0 \\
& \lambda_{2} & \\
& & \ddots \\
0 & &
\end{array}\right]
$$

Let $\left[S_{i j}\right]$ and $\left[X_{i j}\right]$ be the matrix representations of $S$ and $X$ with respect to the above decomposition of $H$. Then $N X-X N=\left[\left(\lambda_{i}-\lambda_{j}\right) X_{i j}\right]$, and in view of the assumption $S \in\{N\}^{\prime}$ we have $S_{i j}=0$ for $i \neq j$. Therefore,

$$
N X-X N+S=\left[\begin{array}{cccc}
S_{11} & & & * \\
& S_{22} & \\
& & \ddots & \\
& & &
\end{array}\right] .
$$

Since $\delta_{N}(X)+S \in J_{\|\| \cdot\|\| \mid}$ and since the norm of an operator matrix always dominates the norm of its diagonal part (see $[9$, p. 82]), it follows that

$$
S \in J_{\|\| \cdot\|\|} \text { and }\left\|\delta_{N}(X)+S|\|\geq\|||S|\right\| \text {. }
$$

Lemma 2. Let $N \in B(H)$ be normal, and set $H_{1}=\bigvee_{\lambda \in \mathbb{C}} \operatorname{ker}(N-\lambda)$. If $S \in\{N\}^{\prime}$ and there is an $X \in B(H)$ such that $\delta_{N}(X)+S \in C_{\infty}$, then $H_{1}$ reduces $S$ and $S \mid H_{1}^{\perp}=0$.

Proof. Since $N$ is normal, $H_{1}$ reduces $N$ and $N \mid H_{1}$ is a diagonal operator. By Fuglede's theorem (see [10, p. 104]) $S^{*} \in\{N\}^{\prime}$, so $H_{1}$ also reduces $S$. Let

$$
N=\left[\begin{array}{cc}
N_{1} & 0 \\
0 & N_{2}
\end{array}\right], \quad S=\left[\begin{array}{cc}
S_{1} & 0 \\
0 & S_{2}
\end{array}\right], \quad X=\left[\begin{array}{ll}
X_{11} & X_{12} \\
X_{21} & X_{22}
\end{array}\right]
$$

on $H=H_{1} \oplus H_{2}$, where $H_{2}=H_{1}^{\perp}$. The assumption $\delta_{N}(X)+S \in C_{\infty}$ implies $\delta_{N_{2}}\left(X_{22}\right)+S_{2} \in C_{\infty}$. Anderson's result (2) (applied to the Calkin algebra $\left.B\left(H_{2}\right) / C_{\infty}\right)$ insures that $S_{2} \in C_{\infty}$. Since the normal operator $N_{2}$ has no eigenvalues and since the compact selfadjoint operator $S_{2}^{*} S_{2}$ belongs to $\left\{N_{2}\right\}^{\prime}$, it follows that $S_{2}^{*} S_{2}=0$. Hence $S_{2}=0$, as desired.

Now we are in a position to prove the main result of this paper.

Theorem 1. Let $N \in B(H)$ be normal, $S \in\{N\}^{\prime}$, and $X \in B(H)$. If $\delta_{N}(X)+$ $S \in J_{\|\| \cdot \| \mid}$, then $S \in J_{\|\| \cdot \|||}$ and

$$
\|\left|\delta_{N}(X)+S\right||| \geq|||S||| .
$$

Proof. Since $\delta_{N}(X)+S \in J_{\|\| \cdot \|} \subseteq C_{\infty}$, it follows by Lemma 2 that on $H=$ $H_{1} \oplus H_{1}^{\perp}$,

$$
N=\left[\begin{array}{cc}
N_{1} & 0 \\
0 & N_{2}
\end{array}\right] \text { and } S=\left[\begin{array}{cc}
S_{1} & 0 \\
0 & 0
\end{array}\right]
$$


where $H_{1}=\bigvee_{\lambda \in \mathbb{C}} \operatorname{ker}(N-\lambda)$. If

$$
X=\left[\begin{array}{ll}
X_{11} & X_{12} \\
X_{21} & X_{22}
\end{array}\right]
$$

on $H=H_{1} \oplus H_{1}^{\perp}$, then

$$
\delta_{N}(X)+S=\left[\begin{array}{cc}
\delta_{N_{1}}\left(X_{11}\right)+S_{1} & * \\
* & *
\end{array}\right] .
$$

Since $\delta_{N}(X)+S \in J_{|\|| \cdot||}$, it follows that $\delta_{N_{1}}\left(X_{11}\right)+S_{1} \in J_{\|||} \cdot \| \mid$. But $N_{1}$ is diagonal and $S_{1} \in\left\{N_{1}\right\}^{\prime}$. Thus, by Lemma $1, S_{1} \in J_{\|\| \cdot\|\|}$ and $\| \mid \delta_{N_{1}}\left(X_{11}\right)+$ $S_{1}\|\| \geq\left\|S_{1}\right\| \|$. Consequently, $S \in J_{\|\| \cdot\|\|}$ and $\left\|\mid \delta_{N}(X)+S\right\|\|\geq\| \delta_{N_{1}}\left(X_{11}\right)+$ $S_{1}\|\geq \geq\| S_{1}|\||=|||S| \mid$.

At the end of this section we use a familiar device of considering $2 \times 2$ operator matrices to extend Theorem 1 to generalized normal derivations.

Corollary 1. Let $N, M, S \in B(H)$ such that $N$ and $M$ are normal and $N S=$ $S M$. If $X \in B(H)$ such that $\delta_{N, M}(X)+S \in J_{|\|\cdot\|| \mid}$, then $S \in J_{\|\| \cdot \| \mid}$ and

$$
\left\|\left|\delta_{N, M}(X)+S\right|\right\| \geq\||| S \mid\| \text {. }
$$

Proof. On $H \oplus H$ consider the operators $L, T$, and $Y$ defined as

$$
L=\left[\begin{array}{cc}
N & 0 \\
0 & M
\end{array}\right], \quad T=\left[\begin{array}{cc}
0 & S \\
0 & 0
\end{array}\right], \quad Y=\left[\begin{array}{cc}
0 & X \\
0 & 0
\end{array}\right] .
$$

Then $L$ is normal, $T \in\{L\}^{\prime}$, and

$$
\delta_{L}(Y)+T=\left[\begin{array}{llll}
0 & \delta_{A, B}(X) & + & S \\
0 & & 0
\end{array}\right] .
$$

Thus by Theorem 1 applied to the operators $L, T$, and $Y$ we have $T \in J_{\|\|} \cdot \|$ and $\left\|\left|\delta_{L}(Y)+T\right|\right\| \geq\||| T \mid\|$. Therefore $S \in J_{|\|\cdot\|| \mid}$ and $\left\|\left|\delta_{A, B}(X)+S\right|\right\| \geq\|S \mid\|$, as desired.

\section{NONNORMAL DERIVATIONS}

Extensions of (3) to certain subnormal operators have been given in [11, Theorems 2 and 3]. In the same vein we devote this section to the extension of the results in $\S 2$ to classes of operators larger than that of normal operators.

Recall that an operator $A \in B(H)$ is called dominant (see [15]) if

$$
\operatorname{ran}(A-\lambda) \subseteq \operatorname{ran}(A-\lambda)^{*} \text { for all } \lambda \in \mathbb{C} .
$$

In view of [6], $A$ is dominant if and only if for each $\lambda \in \mathbb{C}$ there exists a constant $M_{\lambda}$ such that

$$
\left\|(A-\lambda)^{*} f\right\| \leq M_{\lambda}\|(A-\lambda) f\| \text { for all } f \in H .
$$

If there is a constant $M$ such that $M_{\lambda} \leq M$ for all $\lambda \in \mathbb{C}$, then $A$ is called $M$-hyponormal. If $M=1$, then $A$ is hyponormal.

Our promised generalization of (9) can be stated as follows. 
Theorem 2. Let $A, B, S \in B(H)$ such that $A$ is dominant, $B^{*}$ is $M$-hyponormal, and $A S=S B$. If $X \in B(H)$ such that $\delta_{A, B}(X)+S \in J_{\| \mid} \cdot \| \mid$, then $S \in J_{\|\| \cdot \|||}$ and

$$
\left\|\delta_{A, B}(X)+S|\|\geq\|||S|\right\| .
$$

Proof. Since the pair $(A, B)$ satisfies the Fuglede-Putnam property, it follows (see [16] or [19]) that $\overline{\operatorname{ran} S}$ (the closure of $\operatorname{ran} S$ ) reduces $A, \operatorname{ker}^{\perp} S$ (the orthogonal complement of $\operatorname{ker} S$ ) reduces $B$, and $A \mid \overline{\operatorname{ran} S}$ and $B \mid \operatorname{ker}^{\perp} S$ are unitarily equivalent normal operators. Then, with respect to the orthogonal decompositions $H=\overline{\operatorname{ran} S} \oplus(\overline{\operatorname{ran} S})^{\perp}$ and $H=\operatorname{ker}^{\perp} S \oplus \operatorname{ker} S, A$ and $B$ can be respectively represented as

$$
A=\left[\begin{array}{cc}
A_{1} & 0 \\
0 & A_{2}
\end{array}\right] \text { and } B=\left[\begin{array}{cc}
B_{1} & 0 \\
0 & B_{2}
\end{array}\right] .
$$

Now assume that the operators $S, X: \operatorname{ker}^{\perp} S \oplus \operatorname{ker} S \rightarrow \overline{\operatorname{ran} S} \oplus(\overline{\operatorname{ran} S})^{\perp}$ have the matrix representations

$$
S=\left[\begin{array}{cc}
S_{1} & 0 \\
0 & 0
\end{array}\right] \text { and } X=\left[\begin{array}{cc}
X_{1} & X_{2} \\
X_{3} & X_{4}
\end{array}\right] \text {. }
$$

Then $A_{1}$ and $B_{1}$ are normal, and $A_{1} S_{1}=S_{1} B_{1}$.

Applying Corollary 1 to the operators $A_{1}, B_{1}, S_{1}$, and $X_{1}$ we see that $S_{1} \in$ $J_{\||| \cdot||}$. Hence $S \in J_{\||\cdot|||}$ and

$$
\begin{aligned}
\left\|\delta_{A, B}(X)+S \mid\right\| & =\left\|||\left[\begin{array}{cc}
\delta_{A_{1}, B_{1}}\left(X_{1}\right)+S_{1} & * \\
* & *
\end{array}\right]\right\| \| \\
& \geq\left\|\delta_{A_{1}, B_{1}}\left(X_{1}\right)+S_{1}|\|\geq\||\left|S_{1}\||=\|S \mid\|,\right.\right.
\end{aligned}
$$

which completes the proof of the theorem.

The usual operator norm version of (12) has been obtained by Elalami [ 7 , Theorem 4.1] using a different method.

We would like to point out here that in view of [16] Theorem 2 is still valid for any pair of operators $(A, B)$ which satisfies the Fuglede-Putnam property, that is, $A^{*} S=S B^{*}$ whenever $A S=S B$, where $S \in B(H)$. For several such pairs, the reader is referred to [4] and references therein.

A closer look at the proof of Theorem 2 (see also [5, Theorem 1]) leads us to show that if $(A, B)$ satisfies the Fuglede-Putnam property and if $S \in C_{2}$ such that $A S=S B$, then for all $X \in B(H)$ we have

$$
\left\|\delta_{A, B}(X)+S\right\|_{2}^{2}=\left\|\delta_{A, B}(X)\right\|_{2}^{2}+\|S\|_{2}^{2} .
$$

This Hilbert space orthogonality result strengthens (3) and [11, Theorem 3].

It has been shown in [11, Theorem 4] that if $A \in B(H)$ is a cyclic subnormal operator and if $S \in C_{2} \cap\{A\}^{\prime}$, then for all $X \in B(H)$ we have

$$
\left\|\delta_{A}(X)+S\right\|_{2}^{2}=\left\|\delta_{A}(X)\right\|_{2}^{2}+\|S\|_{2}^{2} .
$$

In the same direction, it should be noted that the proof of Theorem 2 can be modified to insure that if $A \in B(H)$ is a cyclic subnormal operator and $S \in J_{\||| \cdot||} \cap\{A\}^{\prime}$, then for all $X \in B(H)$ we have

$$
\left\|\delta_{A}(X)+S|| \geq|||S|\right\| .
$$


To verify (15) we need only show that $\overline{\operatorname{ran} S}$ reduces $A$ and $A \mid \overline{\operatorname{ran} S}$ is normal, for then we can follow the arguments in the proof of Theorem 2. Since $S \in\{A\}^{\prime}$ and $A$ is a cyclic subnormal operator, it follows by Yoshino's result [18] that $S$ is also subnormal. This, together with the assumption $S \in$ $J_{\|\| \cdot \| \mid} \subseteq C_{\infty}$, implies that $S$ is in fact normal. Consequently $S \in\left\{A, A^{*}\right\}^{\prime}$, and so $\overline{\operatorname{ran} S}$ reduces $A$. If $T=A S^{*}$, then $T \in\{A\}^{\prime}$ and $T^{*} T-T T^{*}=$ $S\left(A^{*} A-A A^{*}\right) S^{*} \geq 0$ (because $\left.A^{*} A-A A^{*} \geq 0\right)$. Thus $T$ is a compact hyponormal operator, and hence $T$ is normal. Now we have $A A^{*} S=A S A^{*}=$ $A T^{*}=T^{*} A=S A^{*} A=A^{*} A S$, and so $A \mid \overline{\operatorname{ran} S}$ is normal.

In [8] an example is given to show that the cyclicity assumption on $A$ is necessary for (14) to hold. This gives an affirmative answer to a question raised in [11]. The following example, which will also be used later in the paper, is simpler and shorter than the one given in [8].

Example. Let $U$ be the unilateral shift operator of multiplicity one. On $H \oplus H$, let $A=\left[\begin{array}{ll}U & 0 \\ 0 & 0\end{array}\right], S=\left[\begin{array}{ll}0 & 0 \\ P & 0\end{array}\right]$, and $X=\left[\begin{array}{ll}0 & 0 \\ Q & 0\end{array}\right]$, where $P=1-U U^{*}$ and $Q=$ $P U^{*}$. Then $A$ is a noncyclic subnormal operator, $S \in\{A\}^{\prime}$, and $\delta_{A}(X)+S=$ 0 , yet $\left\|\delta_{A}(X)|\|=\|| S \mid\right\|=1$ for every unitarily invariant norm $\||\cdot \||$.

This example also indicates that Anderson's result (2) cannot be extended to derivations induced by subnormal operators. However, if we require $S$ to be normal, then in this case (2) works for several classes of operators. The list includes compact operators, dominant operators, quasinilpotent operators with positive real parts, and operators $A$ for which $p(A)=0$ for some quadratic polynomial $p$ (see $[7,17]$ ).

Another interesting class of operators for which (2) is true when $S$ is normal is the class of operators $A$ such that $A^{*} A$ and $A+A^{*}$ commute. It is well known that this class enjoys the property that $\|A\|=r(A)$ (the spectral radius of $A$ ) (see [3]). Hence it is elementary to verify that (see [10, p. 130]) for all $X \in B(H)$ and all $\lambda \in \mathbb{C}$ we have

$$
\left\|\delta_{A}(X)+\lambda\right\| \geq|\lambda| .
$$

Based on (16) and the spectral theorem for normal operators it can be shown that if $A, S \in B(H)$ such that $A^{*} A$ commutes with $A+A^{*}, S$ is normal, and $S \in\{A\}^{\prime}$, then for all $X \in B(H)$ we have

$$
\left\|\delta_{A}(X)+S\right\| \geq\|S\| .
$$

To prove (17) we first assume that $S$ is a normal operator with finite spectrum. Then we use a continuity argument to establish the general case.

Finally, we remark that the example presented above shows that (2) fails to hold for an arbitrary (not necessarily normal) operator $S$ in the commutant of $A$.

\section{ACKNOWLEDGMENT}

The author thanks Professor K. Takahashi for his helpful suggestions.

\section{REFERENCES}

1. J. H. Anderson, On normal derivations, Proc. Amer. Math. Soc. 38 (1973), 135-140.

2. R. Bhatia and F. Kittaneh, Norm inequalities for partitioned operators and an application, Math. Ann. 287 (1990), 719-726. 
3. S. L. Campbell and R. Gellar, Linear operators for which $T^{*} T$ and $T^{*}+T$ commute, Proc. Amer. Math. Soc. 60 (1976), 197-202.

4. B. P. Duggal, On generalised Putnam-Fuglede theorems, Monatsh. Math. 107 (1989), 309-332.

5. $\_$A remark on normal derivations of Hilbert-Schmidt type, Monatsh. Math. 112 (1991), 265-270.

6. R. G. Douglas, On majorization, factorization and range inclusion of operators on Hilbert space, Proc. Amer. Math. Soc. 17 (1966), 413-415.

7. S. N. Elalami, Opérateurs complètement finis, preprint.

8. W. Feng and G. Ji, A counterexample in the theory of derivations, Glasgow Math. J. 31 (1989), 161-163.

9. I. C. Gohberg and M. G. Krein, Introduction to the theory of linear nonselfadjoint operators, Transl. Math. Monographs, vol. 18, Amer. Math. Soc., Providence, RI, 1969.

10. P. R. Halmos, A Hilbert space problem book, Springer-Verlag, New York, 1982.

11. F. Kittaneh, On normal derivations of Hilbert-Schmidt type, Glasgow Math. J. 29 (1987), 245-248.

12. P. J. Maher, Commutator approximants, Proc. Amer. Math. Soc. 115 (1992), 995-1000.

13. R. Schatten, Norm ideals of completely continuous operators, Springer-Verlag, Berlin, 1960.

14. B. Simon, Trace ideals and their applications, Cambridge Univ. Press, London and New York, 1979.

15. J. G. Stampfli and B. L. Wadhwa, An asymmetric Putnam-Fuglede theorem for dominant operators, Indiana Univ. Math. J. 25 (1976), 359-365.

16. K. Takahashi, On the converse of the Fuglede-Putnam theorem, Acta Sci. Math. (Szeged) 43 (1981), 123-125.

17. J. P. Williams, Finite operators, Proc. Amer. Math. Soc. 26 (1970), 129-136.

18. T. Yoshino, Subnormal operators with a cyclic vector, Tôhoku Math. J. 21 (1969), 47-55.

19. T. Yoshino, Remarks on the generalized Putnam-Fuglede theorem, Proc. Amer. Math. Soc. 95 (1985), 571-572.

Department of MATHEMATICS, UNiversity OF JoRdan, AMman, JoRDAN 